\title{
Bone health effects of androgen-deprivation therapy and androgen receptor inhibitors in patients with nonmetastatic castration-resistant prostate cancer
}

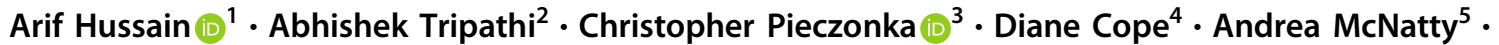 \\ Christopher Logothetis ${ }^{6} \cdot$ Theresa Guise $^{7}$
}

Received: 1 May 2020 / Revised: 24 August 2020 / Accepted: 24 September 2020 / Published online: 7 October 2020

(c) The Author(s) 2020. This article is published with open access

\begin{abstract}
Background Osteoporosis is a skeletal disorder characterized by compromised bone strength, resulting in increased fracture risk. Patients with prostate cancer may have multiple risk factors contributing to bone fragility: advanced age, hypogonadism, and long-term use of androgen-deprivation therapy. Despite absence of metastatic disease, patients with nonmetastatic castrate-resistant prostate cancer receiving newer androgen receptor inhibitors can experience decreased bone mineral density. A systematic approach to bone health care has been hampered by a simplistic view that does not account for heterogeneity among prostate cancer patients or treatments they receive. This review aims to raise awareness in oncology and urology communities regarding the complexity of bone health, and to provide a framework for management strategies for patients with nonmetastatic castrate-resistant prostate cancer receiving androgen receptor inhibitor treatment.

Methods We searched peer-reviewed literature on the PubMed database using key words "androgen-deprivation therapy," "androgen receptor inhibitors," "bone," "bone complications," and "nonmetastatic prostate cancer" from 2000 to present.

Results We discuss how androgen inhibition affects bone health in patients with nonmetastatic castrate-resistant prostate cancer. We present data from phase 3 trials on the three approved androgen receptor inhibitors with regard to effects on bone. Finally, we present management strategies for maintenance of bone health.

Conclusions In patients with nonmetastatic castrate-resistant prostate cancer, aging, and antiandrogen therapy contribute to bone fragility. Newer androgen receptor inhibitors were associated with falls or fractures in a small subset of patients. Management guidelines include regular assessment of bone density, nutritional guidance, and use of antiresorptive bone health agents when warranted.
\end{abstract}

$\triangle$ Arif Hussain

ahussain@som.maryland.edu

1 University of Maryland Greenebaum Cancer Center and Baltimore VA Medical System, Baltimore, MD, USA

2 University of Oklahoma Health Sciences Center, Oklahoma City, OK, USA

3 AMP Urology, Syracuse, NY, USA

4 Florida Cancer Specialists and Research Institute, Fort Myers, FL, USA

5 Mayo Clinic AZ, Scottsdale, AZ, USA

6 MD Anderson Cancer Center, Houston, TX, USA

7 Indiana University School of Medicine, Indianapolis, IN, USA

\section{Introduction}

Osteoporosis is a skeletal disorder that compromises bone strength and increases the risk of fractures [1]. Broadly, in patients with prostate cancer, the interplay of multiple risk factors contributes to bone fragility. Of particular interest, risk factors associated with fragility fracture include hypogonadism; low body weight; current smoking; alcohol intake; vitamin D deficiency; low calcium intake; and longterm use of certain medications, such as glucocorticoids, anticoagulants, anticonvulsants, aromatase inhibitors, cancer chemotherapeutic drugs, and gonadotropin-releasing hormone agonists or antagonists [2]. Compared with a $13 \%$ risk for healthy men $>50$ years old, patients with prostate cancer have a $21-37 \%$ increased risk of fracture [3]. This increased risk may be accounted for by the compounding risk factors contributing to bone fragility and those 
associated with loss of bone mineral density (BMD), such as noted above [4]. Widespread use of androgen-deprivation therapy (ADT) contributes to a high prevalence of osteoporosis in up to $53 \%$ of men with prostate cancer [5]. Bone fragility in patients with metastases has been studied extensively; these studies have led to two US Food and Drug Administration (FDA) approvals, for zoledronic acid and denosumab, for the prevention of metastasisrelated skeletal-related events (SREs) in this particularly high-risk population $[4,6,7]$. Distinct from metastasisrelated SREs, fragility fractures can occur in patients independent of prostate cancer therapy, and thereby decrease patient quality of life [8].

Bone fragility and associated complications can also occur in the absence of documented metastases. Patients with nonmetastatic prostate cancer (nmPC) who receive continuous ADT have been reported to experience significant cumulative loss of BMD: $3.3 \pm 0.7 \%$ in the lumbar spine, $2.1 \pm 0.6 \%$ in the trochanter, and $1.8 \pm 0.4 \%$ in the hip over 12 weeks [9]. BMD loss of 1.4-4.6\% in the lumbar spine, $0.6-3.3 \%$ in the total hip, and $0.7-3.9 \%$ in the femoral neck annually among such patients has also been reported [10]. This translates into fractures as shown in a study of bone complications in 179,744 patients with prostate cancer (metastatic and nonmetastatic) who received ADT [11]. The study revealed that the hazard ratio (HR) for any fracture was 1.4 (95\% CI 1.28-1.53), for hip fracture $1.38(0.20-1.58)$, and for nonskeletal injury 1.01 $(0.90-1.13)$. For patients not on ADT, these values were 0.97 (0.90-1.05), 0.95 (0.84-1.07), and 0.84 (0.77-0.92), respectively [11]. The Prostate Cancer Outcomes Study (PCOS) further explored the relationship between ADT duration and bone complications in a cohort of 3533 patients with nmPC who completed 15-year follow-up surveys to report development of fracture and use of bonerelated medications [12]. Compared with untreated patients, those who received prolonged ADT ( $>1$ year) had a higher odds of fracture (odds ratio [OR] 2.5, 95\% CI 1.1-5.7), BMD testing (OR 5.9, 95\% CI 3.0-12), and bone medication use (OR 4.3, 95\% CI 2.3-8.0). The findings of the PCOS reflect the proportion of patients with nmCRPC (nonmetastatic castrate-resistant prostate cancer) who suffered bone complications during prolonged treatment with ADT [12].

In another study, patients with prostate cancer treated with ADT had an increased risk of any fracture (OR 2.83 [95\% CI 2.52-3.17]) and of hip fracture requiring hospitalization (OR 1.82 [95\% CI 1.44-2.30]) [13]. Excluding pathological fractures and spinal cord compression reduced the calculated overall OR to 1.47 , still an elevated risk from ADT use [13]. Among the bone markers studied that are associated with increased osteoclastic bone destruction, Ntelopeptide has been shown to be the most significant predictor of death in patients with prostate cancer (relative risk [RR] 3.25; 95\% CI 2.26-4.68) and is further evidence that more bone destruction increases the risk of death [14]. Close monitoring of bone health for patients on ADT has been recommended, as fractures increase morbidity and risk of mortality when they occur [13].

Novel and more potent androgen receptor inhibitors (ARIs) are increasingly used in earlier disease settings, resulting in longer exposure times that, in turn, can result in increased risk of falls and fractures. The degree of increase risk of falls and fractures is different for the novel ARIs [15-17]. Collectively, these observations raise the concern that the increasingly prevalent use of second-generation antiandrogens in earlier disease states may increase the number of patients at risk for fracture. In older adults, increased fracture incidence also plays a role in decreased quality of life [8].

Identification and management of fragility fracture risk prior to actual fracture is important to the care of patients with prostate cancer. A systematic approach to bone health care for patients with prostate cancer has been hampered by a lack of standardized guidelines, lack of BMD testing, and gaps in education about: (a) adverse events (AEs) associated with ADTs, (b) diet and lifestyle on bone health, and (c) pharmacologic intervention to reduce fracture risk [4]. The purpose of this review is to raise awareness in the oncology and urology communities about the importance of bone health among patients with prostate cancer eligible for treatment with ARIs, to discuss the determinants of risk, and particularly to highlight emerging data about the management of fracture risk in patients. The review was prompted by the potential increased risk of bone complications in patients benefiting from newly approved life prolonging therapies that also increase bone fragility. The goal of the review is to provide a blueprint for developing multidisciplinary management strategies for improving bone health for patients with nmCRPC.

\section{Pathophysiology of bone fragility and changes due to aging and prostate cancer}

In patients with prostate cancer, three main drivers of fragility fracture risk are: (1) the aging process, (2) cancer treatment, primarily with ADT, and (3) cancer phenotype. Normally, peak BMD is achieved in young adulthood, generally around 20 years of age; it is maintained until $\sim 40$ years of age, and then declines for the reminder of one's lifespan (Fig. 1) [18-20]. Fracture risk rises substantially with age to a degree that outpaces the effects attributed to loss of BMD [18]. Cancer phenotype refers to the variation between individuals with the same cancer diagnosis. 


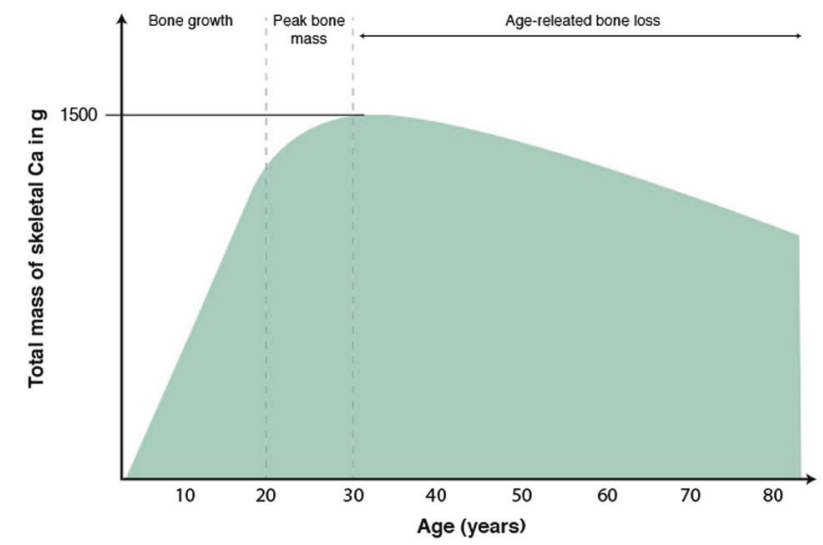

Fig. 1 Adapted from [19] and [20] to show age-related bone loss in men. Santos et al. [19] is an open access article distributed under the terms of the Creative Commons CC BY license, which permits unrestricted use, distribution, and reproduction in any medium.

Several pathways are involved in age-related bone loss among men within the general population. A major contributor to BMD loss with aging is testosterone deficiency due to increases in sex hormone-binding globulin, which reduces free testosterone [18]. Testosterone has several effects on bone cells (osteoblasts, osteoclasts, and osteocytes), all of which express androgen receptors. Ligand binding of the androgen receptor stimulates osteoblast proliferation and suppresses apoptosis [21]. Androgen receptor activation by dihydrotestosterone of osteoclasts inhibits bone resorption in in vitro studies [21]. Decreased testosterone also leads to increased osteoblast-secreted RANKL (receptor activator of nuclear factor kappa-B ligand) levels, which then stimulates osteoclast-mediated bone resorption [21]. Another pathway to bone loss is the accumulation of impaired osteoblasts due to age-related telomere shortening, accumulation of oxidative stress, and DNA damage, all of which can lead to inefficient bone formation and remineralization [18].

In patients with prostate cancer, including nmCRPC, certain androgen-inhibiting treatments can contribute to bone fragility by causing accelerated osteoclastic bone destruction [4, 8]. Although rare, tumor-induced osteomalacia (TIO) may be involved as well. TIO is associated with increased production of fibroblast growth factor (FGF)-23, which results in impaired bone mineralization by inappropriate renal phosphate wasting, reduced calcium absorption and marked softening of bones [22]. Osteomalacia in general can be caused by insufficient calcium, phosphate or vitamin $\mathrm{D}$ and is associated with reduced bone density, but can be distinguished from osteoporosis by bone biopsy. Chronic vitamin D deficiency can also contribute to overall skeletal morbidity [22].

FGF signaling in general provides a positive feedback loop between bone cells and the tumor microenvironment of prostate cancer cells. In particular, blockade of the FGF-1 receptor in osteoblasts can partially mediate the antitumor effect of the vascular-endothelial growth factor inhibitor, dovitinib, a drug that also inhibits FGF-1 and FGF3 signaling [23]. Moreover, FGF-23 is a downstream target of FGF-1 signaling in osteocytes; therefore, blocking FGF-1 blocks FGF-23 [23]. A proof-of-principle study in patients with metastatic castration-resistant prostate cancer (mCRPC) showed that treatment with dovitinib reduced bone metastases: $26 \%$ of patients showed improvement on bone scan, with a median treatment duration of 19.9 weeks [23].

Additional considerations that contribute to bone fragility are the effects of androgen-deprivation and aging that decrease muscle mass and function, increase fat, and reduce mobility $[24,25]$. Testosterone plays a key role in the preservation of muscle mass [24], whereas ADT contributes to muscle dysfunction and loss of muscle mass. In a study of 32 evaluable patients treated with gonadotropic releasing hormone agonists over 48 weeks, lean body mass decreased by $2.7 \%(P<0.001)$; at the same time, body mass index increased $2.4 \%(P=0.005)$ [26]. Untreated age-related muscle wasting may contribute to falls and fractures. Among persons $\geq 65$ years, $5-13 \%$ have loss of muscle mass, and this proportion increases to $50 \%$ for those $>80$ years of age [25]. Mobility is also integral to overall bone health; acute immobilization has been shown to accelerate bone turnover and bone loss which, in turn, contribute to falls and fractures in the elderly. These interdependent effects on fracture risk: bone fragility, muscle weakness, and increased fall risk emphasize the need to carefully select therapeutic interventions in the context of underlying bone health. It should be noted that many of the above pathophysiologic processes associated with bone fragility are likely to be operative to varying degrees in patients with either nmCRPC or mCRPC.

\section{ARIs in nmCRPC and effects on bone: insights from clinical trial data}

The approved next generation ARIs-apalutamide, darolutamide, and enzalutamide - have demonstrated efficacy in patients with nmCRPC in the SPARTAN, ARAMIS, and PROSPER phase 3 trials respectively; each trial also evaluated potential risk of falls and fractures [15-17]. These three agents were each added to ADT and evaluated against placebo in similarly designed studies of nmCRPC with prostate-specific antigen doubling time (PSADT) $\leq 10$ months, with the primary endpoint being metastasisfree survival. Patients in these three trials were stratified by use of bone-sparing agents (not specified) at baseline, along with other criteria. Each trial favored addition of ARI to 
Table 1 Falls, fractures, and other bone-related AEs in phase 3 trials for ARIs.

\begin{tabular}{|c|c|c|c|c|c|c|c|}
\hline \multirow[b]{2}{*}{ Study } & \multirow[b]{2}{*}{ Drug } & \multicolumn{3}{|c|}{ Treatment arm } & \multicolumn{3}{|l|}{ Placebo arm } \\
\hline & & Falls $^{\mathrm{a}} n(\%)$ & Fractures $n(\%)$ & Other $^{\mathrm{b}} n(\%)$ & Falls $^{\mathrm{a}} n(\%)$ & Fractures $n(\%)$ & Other $^{\mathrm{b}} n(\%)$ \\
\hline SPARTAN $(N=1207)[15]$ & Apalutamide & $125(15.6)$ & $94(11.7)$ & NR & $36(9.0)$ & $26(6.5)$ & NR \\
\hline ARAMIS $(N=1509)$ [17] & Darolutamide & $40(4.2)$ & $40(4.2)$ & $139(14.6)$ & $26(4.7)$ & $20(3.6)$ & $68(12.2)$ \\
\hline PROSPER $(N=1401)[16,28]$ & Enzalutamide & $106(11)$ & $91(10)$ & $73(8)$ & $19(4)$ & $23(5)$ & $33(7)$ \\
\hline
\end{tabular}

$A E$ adverse event, $A R I$ androgen receptor inhibitor, $N R$ not reported.

${ }^{a}$ In SPARTAN, falls were deemed treatment-related by the investigators. In ARAMIS, falls included events recorded as accidents, and were determined to have been accidental falls.

${ }^{b}$ Other includes back pain in PROSPER, and back pain or pain in an extremity in ARAMIS.

ADT in terms of statistically significant improvement in metastasis-free survival [15-17]. Updated data from the ARI trials also showed increase in overall survival compared with placebo (HR for apalutamide 0.78 [95\% CI 0.64-0.96; $P=0.0161]$ ) [27]; HR for darolutamide 0.69 [95\% CI 0.53-0.88; $P<0.003$ ] [28]; HR for enzalutamide 0.73 [95\% CI $0.61-0.89 ; P=0.001]$ [29].

Although comparisons across clinical trials are imprecise, the similar designs and placebo-control arms allow for some comparison of these agents to be made. SPARTAN $(N=1207)$ evaluated the efficacy of apalutamide in men with nmCRPC who had PSADT of $\leq 10$ months [15]. In SPARTAN, 1207 enrolled patients were randomized to receive apalutamide or placebo. Falls and fractures occurred in $125(15.6 \%)$ and $94(11.7 \%)$ patients in the apalutamide arm, compared with $36(9.0 \%)$ and $26(6.5 \%)$ patients in the placebo arm, respectively (Table 1) [15]. A secondary analysis of SPARTAN data showed that patients $\geq 75$ years of age had significantly higher risk of fractures and falls (HR 1.8, 95\% CI 1.3-2.7), compared with patients $<75$ years of age [30].

In ARAMIS $(N=1509)$, which evaluated the efficacy and safety of darolutamide in men with nmCRPC and a PSADT of $\leq 10$ months against placebo, AEs that occurred in the darolutamide arm included back pain (84 patients, $8.8 \%$ ), pain in an extremity (55 patients, $5.8 \%$ ), fractures (40 patients, $4.2 \%$ ) and falls (40 patients, $4.2 \%$ ) (Table 1) [17]. For patients who received placebo, back pain occurred in $50(9.0 \%)$ patients, pain in an extremity occurred in 18 (3.2\%), fractures in $20(3.6 \%)$. patients and falls in 26 (4.7\%) patients [17]. Although no secondary analysis or additional trial data for darolutamide are yet available, these data suggest that darolutamide may not significantly increase fracture incidence beyond any background risk.

The PROSPER trial $(N=1401)$ compared enzalutamide to placebo in men with nmCRPC and a PSADT of $\leq 10$ months [16]. Among the AEs occurring in $\geq 5 \%$ of patients in the enzalutamide arm, falls and back pain occurred in $106(11 \%)$ and $73(8 \%)$ patients, respectively (Table 1) [16]. For patients who received placebo, falls and back pain occurred in $4 \%$ and $7 \%$, respectively [16]. More fractures were reported for patients who received enzalutamide (10\%) than placebo (5\%) [31].

Another next generation hormonal agent, abiraterone, which primarily inhibits androgen biosynthesis but also has some ARI activity, has been evaluated in the smaller IMAAGEN phase 2 trial in patients with high-risk nmCRPC; it demonstrated a significant (87\%) reduction in PSA level. At 48 months, $62 \%$ of patients were estimated radiographically to be progression-free. However, data on falls and fracture risks were not reported [32].

Comparison across these three trials is problematic; nevertheless, the initial experience from the large ARI phase 3 trials suggest that patients given darolutamide are less susceptible to falls and similar to placebo. Additional realworld experience will help further clarify the role of these next generation antiandrogen receptor targeting agents in terms of relative fracture and fall risks.

\section{Management strategies}

The latest guidelines addressing bone health-related risks among prostate cancer patients on ADT are not applied uniformly in practice $[4,6,10]$. Thus, there is an unmet need for the oncology and urology communities to address bone health management, including in patients with nmCRPC, a disease state in which newer androgen receptor targeting agents are being increasingly incorporated in addition to standard ADT as part of patients' overall treatment. Management strategies are summarized in Table 2.

\section{Screening}

Bone density screening is essential for early detection of patients at risk for SREs, but is not performed consistently in men [33]. Results of a study by the US Department of Veterans Affairs suggest that routine screening is warranted among patients with prostate cancer (Table 2) [33]. 


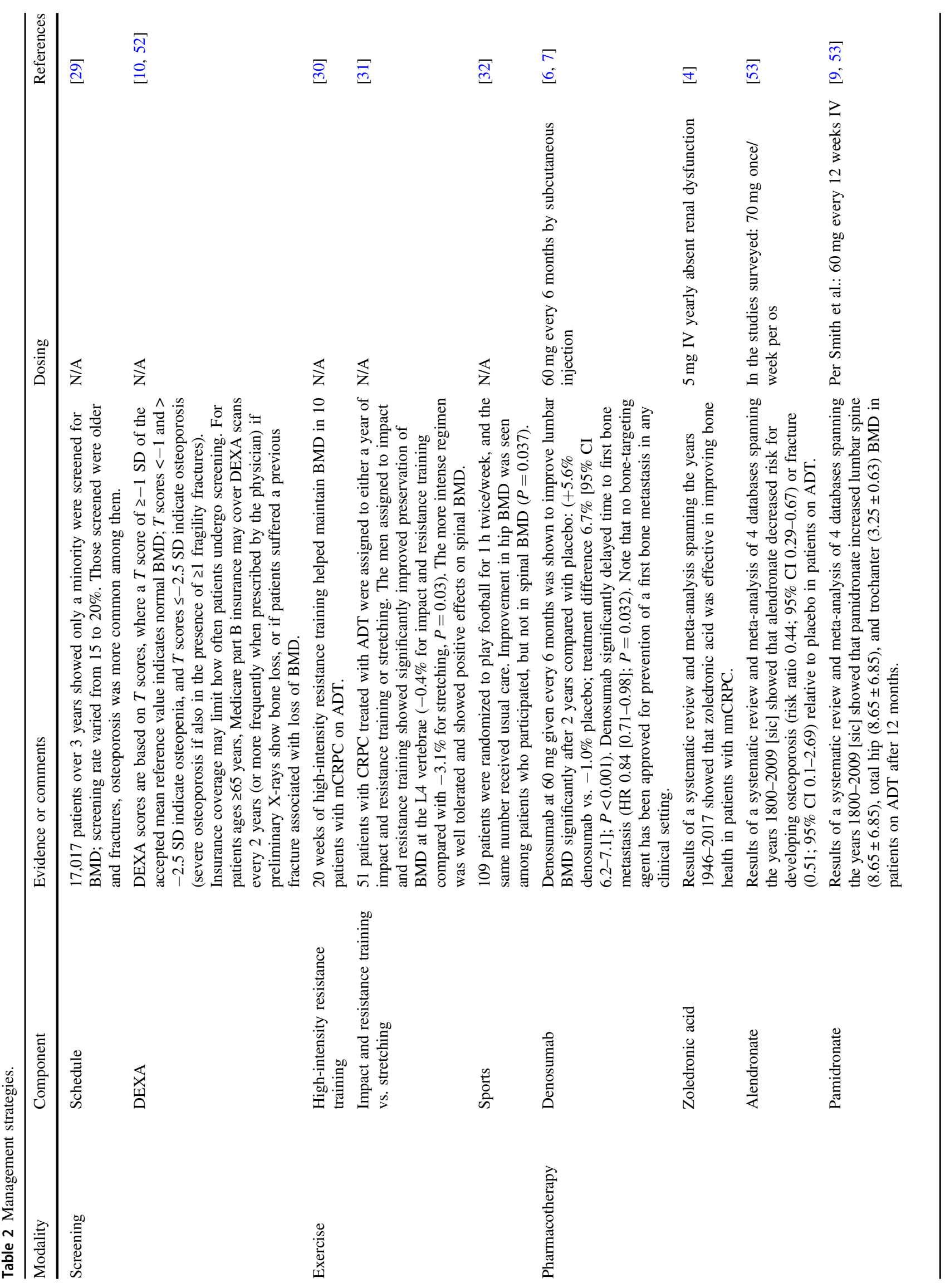




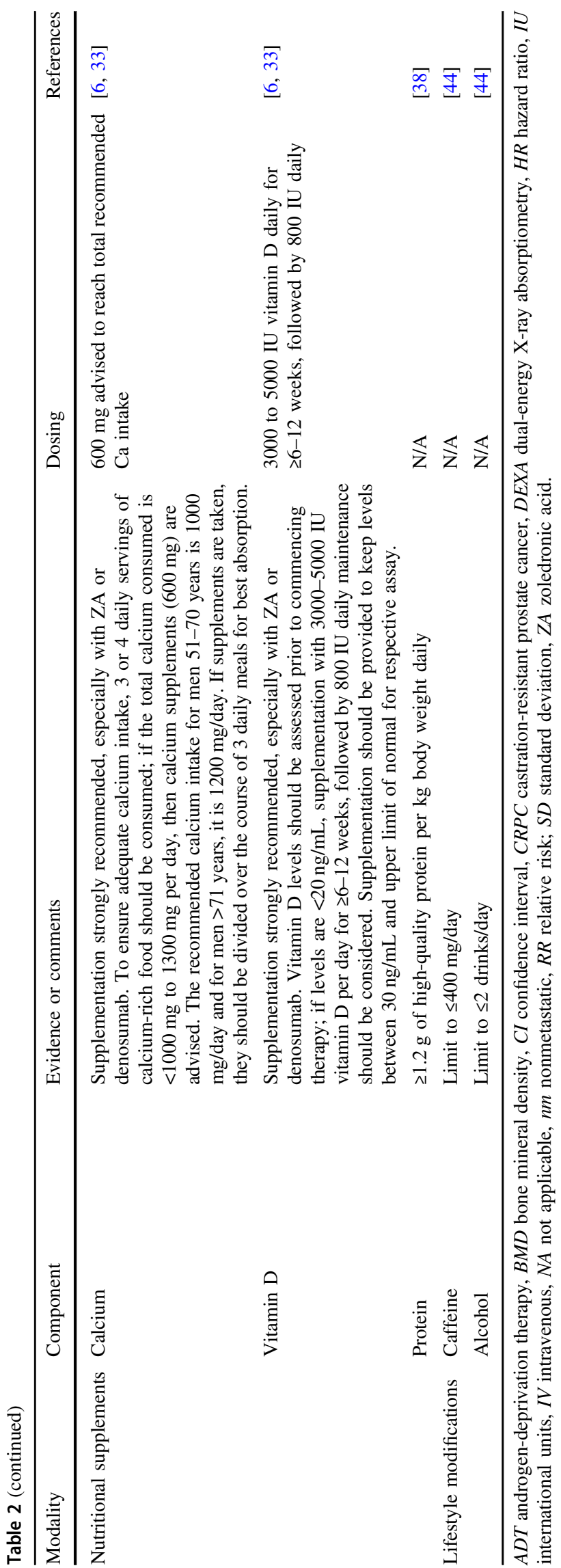

Dual-energy X-ray absorptiometry (DEXA) is the most widely used method to measure BMD. The World Health Organization (WHO) and many national health agencies recommend that BMD be monitored in patients with prostate cancer (Table 2) [10]. As per WHO guidelines, baseline DEXA scans are recommended. Although computed tomography and other imaging techniques might be used to assess bone mineralization, the widespread application to clinical practice is limited for these modalities [10].

\section{Exercise}

Exercise has benefits in the maintenance of bone health during aging. During weight-bearing exercise, mechanosensors, such as stretch-activated ion channels, within osteocytes can trigger cascades leading to new bone deposition [19].

The effect of exercise in patients with nmCRPC treated with ARIs has not yet been studied, but some conclusions about the efficacy of exercise on preserving bone health may be drawn from prior studies on patients with localized prostate cancer. In one small study, patients who underwent high-intensity resistance-training maintained BMD, although none showed an increase in BMD (Table 2) [34]. In another study, patients with prostate cancer treated with ADT were assigned to either a year of impact and resistance training or stretching. The more intense training regimen was tolerated well by patients receiving ADT and showed positive effects on spinal BMD (Table 2) [35]. Recently, investigators evaluated sports participation as effective exercise for preservation of BMD in individuals with prostate cancer, of whom a proportion were on ADT [36]. Among the patients on ADT, there was statistically significant improvement in hip BMD among patients who participated in sports (Table 2), but not in spinal BMD [36]. A prospective trial to determine the effect of exercise among patients with prostate cancer treated with ADT has recently been completed [37]. This was a year-long randomized controlled study comparing exercise or nutritional supplementation (calcium, vitamin $\mathrm{D} ; n=51$ ) versus no intervention (usual care group; $n=51$ ) [37]. Exercise consisted of aerobic exercise, resistance training, and weight-bearing exercise [37]. Assessments of BMD were made by DEXA scan and bone strength determinations by peripheral quantitative computed tomography [37]. The trial has been completed and, as of this writing, final data are awaited.

Safe movement and exercise programs may be developed under the guidance of physical therapists for patients were warranted. As well, some clinicians may also recommend home resistance training programs. 


\section{Pharmacotherapy}

Pharmacotherapy for maintaining or improving bone health includes bone resorption inhibitors, bisphosphonates, and denosumab. Although the ultimate target for bone-protecting/bone-sparing agents (BPA) is the osteoclast, they differ in their mechanism of action and pharmacokinetics, which have important clinical implications. Bisphosphonates (i.e., bone mineral analogs) become part of the bone mineral matrix, whereas denosumab (a monoclonal antibody) binds to a cytokine ligand circulating outside bone cells (Table 3) [38]. Bisphosphonates, such as zoledronic acid, inhibit farnesyl pyrophosphate synthase, ultimately leading to osteoclast apoptosis. Zoledronic acid and other bisphosphonates are deposited into mineralized bone matrix, and taken up by bone-resorbing osteoclasts, thereby contributing to their cellular selectivity. The activity of denosumab, in contrast, is directed against RANKL, a cytokine that promotes osteoclast recruitment, maturation, activation, and survival [38]. Clearance of bisphosphonates is through bone turnover and for denosumab through the reticuloendothelial system (Table 3) [38]. The relevance of these BPAs in decreasing fracture risk has been underscored by the recent European Organisation for Research and Treatment of Cancer (EORTC) 1333/PEACE III trial that evaluated enzalutamide plus radium-223 versus enzalutamide alone in patients with asymptomatic/minimally symptomatic mCRPC; use of BPAs 6 weeks prior to initiating radium223 significantly reduced the fracture rate in patients [39]. Although such a formal evaluation of BPAs in nmCRPC patients has not yet been done, data from the PEACE III trial in patients with mCRPC highlight the relevance of BPAs in modifying fracture risk among patients at risk.

Importantly, among patients with nmCRPC, BPAs have also been shown to have value in maintaining bone health [4]. Studies comparing their efficacy in preserving BMD in patients with prostate cancer have shown that intravenous zoledronic acid preserved BMD better than did other bisphosphonates or denosumab. It is important to note that patients in these studies were treated with ADT, and these results may not hold for patients treated with ARIs [40]. Denosumab, available from the manufacturer in a $60-\mathrm{mg}$ formulation given every 6 months, has been shown to increase bone mass in men with $\mathrm{nmPC}$ receiving ADT who are at high risk of fracture (Table 3) [40]. In the three ARI trials discussed above, at baseline Fizazi et al. reported 3\% (darolutamide) vs. 6\% (placebo) of patients used bonesparing agents [17], Smith et al. reported $10.2 \%$ vs. 9.7\% [15], Hussain et al. reported $11 \%$ vs. $10 \%$ [16].

In deciding whether or when to commence use of BPAs, practitioners might assess the FRAX-estimated risk of developing a fragility fracture using femoral BMD [8]. It should be noted that FRAX-derived fracture probability has 
yet to be validated in patients with nmCRPC, and a consensus intervention threshold has not been established [8].

Considering that patients with prostate cancer are likely to be treated with BPAs for longer duration than was investigated in clinical trials, effects of their long-term use should be taken into account when managing bone health [41]. Hypocalcemia has been reported to occur more frequently with denosumab than with zoledronic acid; it can be prevented with adequate calcium and vitamin D supplementation [41]. Recommended supplementation doses for when serum 25-hydroxyvitamin D2 and D3 levels are lower than $30 \mathrm{ng} / \mathrm{mL}$ are outlined in Table 2: if levels are $<20 \mathrm{ng} / \mathrm{mL}$, supplementation with 3000-5000 IU vitamin D per day for at least 6-12 weeks followed by 800 IU daily maintenance should be considered [6, 42]. Vitamin D levels should be monitored every 6 months during drug treatment. A second condition, osteonecrosis of the jaw (ONJ), occurs relatively infrequently among patients with prostate cancer treated with denosumab or zoledronic acid [43]. A composite analysis of patients with breast and prostate cancer treated with these agents showed the incidence of ONJ in the first year to be $1.1 \%$, rising to $2.2 \%$ in the second year, $3.7 \%$ in the third year, and $4.6 \%$ each year thereafter [41]. Good oral hygiene reduces the incidence of $\mathrm{ONJ}$; oral health should be addressed before commencing treatment with bone health agents, and preventive measures may be implemented by a dental health professional if necessary [44]. In particular, invasive dental procedures should be completed, and ill-fitting appliances should be addressed to reduce the incidence of ONJ [6]. For the many patients who present with underlying renal dysfunction, close monitoring for hypocalcemia is warranted. Caution should be taken when prescribing zoledronic acid in patients with reduced renal function; in these patients, doses should be adjusted accordingly. Denosumab does not need dose adjustments in patients with impaired renal function.

Finally, a treatment plan for duration and discontinuation of therapy should be developed. An important, but often not considered, aspect of denosumab treatment is the reported development of rebound osteoclastogenesis, which occurs when denosumab is discontinued [45]. Osteoclastogenesis can result in rapid bone loss and fracture in the year following cessation of denosumab therapy. Such increases in bone destruction can also enrich the local bone microenvironment to fuel cancer growth if tumor cells are present in the bone microenvironment. This poses a theoretical risk for bone metastases. This rapid bone loss does not occur when zoledronic acid is discontinued because the drug has a long half-life in bone. Thus, if denosumab is discontinued, zoledronic acid should be given, administered at a time when bone destruction recurs, to prevent the rapid bone loss. Currently, there are no clinical guidelines on how to cease denosumab treatment [46]. As such, health care providers should consider consultation with a specialist in metabolic bone disease to guide the transition of denosumab to zoledronic acid or another BPA [45, 46]. Currently available data suggest that administering zoledronic acid within 7-8 months or sooner after the last dose of denosumab may be best to minimize bone loss [47].

\section{Nutritional considerations and supplementation}

Guidelines for nutritional supplementation have not yet been developed for nmCRPC treated with ARIs, as these drugs are too recent for specific studies to have been completed. Some insights may be gleaned from prior experience with older forms of androgen inhibition (ADT and antiandrogens), and the use of nutritional supplementation to help mitigate AEs on bone health in patients with prostate cancer. A systematic review of the literature showed that adequate nutrition during ADT may have helped mitigate treatment-related AEs on bone and that patients require guidance to help them follow a diet that will meet their complete nutritional needs [48].

A randomized phase 2 trial of patients with prostate cancer who received ADT examined educational strategies (family physician, bone health support care, or usual care) to assess compliance with methods directed toward improving bone health [49]. Secondary efficacy outcomes included vitamin D (800 mg to 2000 IU daily) and calcium (1000-1200 mg daily) use [48, 49]. Family physician and bone health support care educational strategies were found to improve bone health, including higher compliance for intake of calcium [49]. Extension of similar studies to ARIs might be a future consideration. Vitamin D should be recommended based on patient serum 25-hydroxyvitaminD2 and -D3 levels, calcium intake should be at least 1000 $\mathrm{mg} /$ day (through food and/or supplements), and protein intake should be roughly $75 \mathrm{~g} /$ day [42]. Based on our collective clinical experience, several strategies are proposed to ensure adequate vitamin $\mathrm{D}$, calcium, and protein intake, summarized in Table 2.

\section{Other lifestyle modifications}

The positive effects of pharmacotherapy, exercise, and sufficient calcium and vitamin D intake may be augmented by additional changes to lifestyle. Most common recommendations include limiting caffeine consumption and imbibing only moderate amounts of alcohol [48]. Specific dietary recommendations have not been offered in the literature, although the importance of educating patients about the details outlined above (quantities of caffeine, alcohol, 
importance of regular exercise, and among others) should not be overlooked when discussing bone health with patients [48].

\section{Summary and conclusions}

Older men, including those with prostate cancer, are at increased risk of diseases associated with decreased BMD and increased bone fragility and, consequently, are at increased fracture risk. Treatments that inhibit androgen production and androgen action have been associated with bone loss. For these reasons, the effects of ARI on bone health should be included when discussing treatment options for patients with nmCRPC. Bone health agents may be prescribed for patients at increased risk for fractures. In patients with nmCRPC, aging and androgen-inhibiting treatment both contribute to bone fragility. The increased risk of fractures following androgen-inhibition is due to increased osteoclastic bone destruction in addition to muscle dysfunction and increased risk of fall [4]. Reduced testosterone has a negative impact on muscle mass, which may lead to less mobility, further contributing to decreased BMD [24, 25, 50].

Clinical trial data for the approved ARIs apalutamide, darolutamide, and enzalutamide showed that falls and fractures occurred in a minority of patients with nmCRPC treated with these drugs; of the three, darolutamide was associated with the lowest incidence of falls and fractures (Table 1) [15, 16, 51]. However, this is not to say that one agent is associated with less fracture risk than the others, and as noted above updated data from these trials show that all three ARIs discussed are associated with increased overall survival compared with placebo, providing important treatment options for high-risk nmCRPC patients [27-29].

Management strategies for patients with nmCRPC treated with ARIs should include baseline and regular screening by DEXA [10], and lifestyle modifications, including recommendations for safe movement, and adequate and safe exercise [34, 35]. In addition, bone health agents may be prescribed, either denosumab or a bisphosphonate, both of which have been studied in patients with nmCRPC [4]. Nutritional assessment is important to ensure that patients have adequate intake of protein, calcium, and vitamin D. Caffeine and alcohol should be avoided. A multidisciplinary assessment and approach to management is likely to lead to optimal outcomes, including bone health among patients with nmCRPC.

Acknowledgements Beverly E. Barton, PhD, and D. Michele Nikoloff, $\mathrm{PhD}$, ScioScientific, LLC, assisted with writing and editing. This study was supported in part by Bayer. The authors received no compensation from Bayer HealthCare Pharmaceuticals, Inc. for their participation.

\section{Compliance with ethical standards}

Conflict of interest AH has consulted for: Bayer, AstraZeneca, BristolMyers-Squibb; served on advisory boards: Bayer, Exelixis, Janssen, AstraZeneca, Novartis. AT has advised for: Foundation Medicine, Pfizer; received research funding to institution from: EMD Serono; Aravive, Inc.; WindMIL Therapeutics; Corvus Pharmaceuticals; Clovis Oncology; Bayer. CP is a paid consultant for Dendreon and Janssen; part of the speakers' bureaus for Janssen, Merck, Sun, Bayer, Pfizer, and Astellas; has received research funding from Amgen, Janssen, Merck, AstraZeneca, Pfizer, Astellas, Bayer, Dendreon, and Veru. DC and $\mathrm{AMcN}$ declare no conflicts of interest. CL has received research or grant support from Janssen, Sanofi, BMS, and Pfizer; and honoraria from Amgen, Bayer, and Merck. TAG has been an advisor for Bayer and has received research grant funding from Bayer.

Publisher's note Springer Nature remains neutral with regard to jurisdictional claims in published maps and institutional affiliations.

Open Access This article is licensed under a Creative Commons Attribution 4.0 International License, which permits use, sharing, adaptation, distribution and reproduction in any medium or format, as long as you give appropriate credit to the original author(s) and the source, provide a link to the Creative Commons license, and indicate if changes were made. The images or other third party material in this article are included in the article's Creative Commons license, unless indicated otherwise in a credit line to the material. If material is not included in the article's Creative Commons license and your intended use is not permitted by statutory regulation or exceeds the permitted use, you will need to obtain permission directly from the copyright holder. To view a copy of this license, visit http://creativecommons. org/licenses/by/4.0/.

\section{References}

1. Lorentzon M, Cummings SR. Osteoporosis: the evolution of a diagnosis. J Intern Med. 2015;277:650-61.

2. Qaseem A, Forciea MA, McLean RM, Denberg TD. Clinical Guidelines Committee of the American College of P. Treatment of low bone density or osteoporosis to prevent fractures in men and women: a clinical practice guideline update from the American College of Physicians. Ann Intern Med. 2017;166:818-39.

3. Lassemillante A-CM, Doi SAR, Hooper JD, Prins JB, Wright ORL. Prevalence of osteoporosis in prostate cancer survivors: a meta-analysis. Endocrine. 2014;45:370-81.

4. Alibhai SMH, Zukotynski K, Walker-Dilks C, Emmenegger U, Finelli A, Morgan SC, et al. Bone health and bone-targeted therapies for nonmetastatic prostate cancer: a systematic review and meta-analysis. Ann Intern Med. 2017;167:341-50.

5. Lassemillante A-CM, Doi SAR, Hooper JD, Prins JB, Wright ORL. Prevalence of osteoporosis in prostate cancer survivors II: a meta-analysis of men not on androgen deprivation therapy. Endocrine. 2015;50:344-54.

6. Saylor PJ, Rumble RB, Tagawa S, Eastham JA, Finelli A, Reddy PS, et al. Bone health and bone-targeted therapies for prostate cancer: ASCO endorsement of a cancer care Ontario Guideline. J Clin Oncol. 2020;0:JCO.19.03148.

7. Smith MR, Saad F, Coleman R, Shore N, Fizazi K, Tombal B, et al. Denosumab and bone-metastasis-free survival in men with 
castration-resistant prostate cancer: results of a phase 3, randomised, placebo-controlled trial. Lancet. 2012;379:39-46.

8. Cianferotti L, Bertoldo F, Carini M, Kanis JA, Lapini A, Longo N, et al. The prevention of fragility fractures in patients with nonmetastatic prostate cancer: a position statement by the international osteoporosis foundation. Oncotarget. 2017;8:75646-63.

9. Smith MR, McGovern FJ, Zietman AL, Fallon MA, Hayden DL, Schoenfeld DA, et al. Pamidronate to prevent bone loss during androgen-deprivation therapy for prostate cancer. N Engl J Med. 2001;345:948-55.

10. Lee CE, Leslie WD, Czaykowski P, Gingerich J, Geirnaert M, Lau YKJ. A comprehensive bone-health management approach for men with prostate cancer receiving androgen deprivation therapy. Curr Oncol. 2011;18:e163-72.

11. Wallander M, Axelsson KF, Lundh D, Lorentzon M. Patients with prostate cancer and androgen deprivation therapy have increased risk of fractures-a study from the fractures and fall injuries in the elderly cohort (FRAILCO). Osteoporos Int. 2019;30:115-25.

12. Morgans AK, Fan KH, Koyama T, Albertsen PC, Goodman M, Hamilton AS, et al. Bone complications among prostate cancer survivors: long-term follow-up from the prostate cancer outcomes study. Prostate Cancer Prostatic Dis. 2014;17:338-42.

13. Wang A, Obertová Z, Brown C, Karunasinghe N, Bishop K, Ferguson L, et al. Risk of fracture in men with prostate cancer on androgen deprivation therapy: a population-based cohort study in New Zealand. BMC Cancer. 2015;15:837.

14. Brown JE, Cook RJ, Major P, Lipton A, Saad F, Smith M, et al. Bone turnover markers as predictors of skeletal complications in prostate cancer, lung cancer, and other solid tumors. JNCI J Natl Cancer Inst. 2005;97:59-69.

15. Smith MR, Saad F, Chowdhury S, Oudard S, Hadaschik BA, Graff JN, et al. Apalutamide treatment and metastasis-free survival in prostate cancer. N Engl J Med. 2018;378:1408-18.

16. Hussain M, Fizazi K, Saad F, Rathenborg P, Shore N, Ferreira U, et al. Enzalutamide in men with nonmetastatic, castration-resistant prostate cancer. N Engl J Med. 2018;378:2465-74.

17. Fizazi K, Shore N, Tammela TL, Ulys A, Vjaters E, Polyakov S, et al. Darolutamide in nonmetastatic, castration-resistant prostate cancer. N Engl J Med. 2019;380:1235-46.

18. Khosla S. Pathogenesis of age-related bone loss in humans. J Gerontol A Biol Sci Med Sci. 2013;68:1226-35.

19. Santos L, Elliott-Sale KJ, Sale C. Exercise and bone health across the lifespan. Biogerontology. 2017;18:931-46.

20. Betts JG, Young KA, Wise JA, Johnson E, Poe B, Kruse DH, et al. 6.6 Exercise, nutrition, hormones, and bone tissue. In: anatomy and physiology Houston TX: OpenStax; 2013.

21. Mohamad N-V, Soelaiman I-N, Chin K-Y. A concise review of testosterone and bone health. Clin Inter Aging. 2016;11:1317-24.

22. Minisola S, Peacock M, Fukumoto S, Cipriani C, Pepe J, Tella $\mathrm{SH}$, et al. Tumour-induced osteomalacia. Nat Rev Dis Prim. 2017;3:17044.

23. Wan X, Corn PG, Yang J, Palanisamy N, Starbuck MW, Efstathiou E, et al. Prostate cancer cell-stromal cell crosstalk via FGFR1 mediates antitumor activity of dovitinib in bone metastases. Sci Transl Med. 2014;6:252ra122-252ra122.

24. Saad F, Röhrig G, von Haehling S, Traish A. Testosterone deficiency and testosterone treatment in older men. Gerontology. 2017;63:144-56.

25. Padilla Colón CJ, Molina-Vicenty IL, Frontera-Rodríguez M, García-Ferré A, Rivera BP, Cintrón-Vélez G, et al. Muscle and bone mass loss in the elderly population: advances in diagnosis and treatment. J Biomed. 2018;3:40-9.

26. Smith MR, Finkelstein JS, McGovern FJ, Zietman AL, Fallon MA, Schoenfeld DA, et al. Changes in body composition during androgen deprivation therapy for prostate cancer. J Clin Endocrinol Metab. 2002;87:599-603.
27. Small EJ, Saad F, Chowdhury S, Hadaschik B, Graff JN, Olmos D, et al. SPARTAN, a phase 3 double-blind, randomized study of apalutamide (APA) versus placebo (PBO) in patients (pts) with nonmetastatic castration-resistant prostate cancer (nmCRPC). American Society for Clinical Oncology: Chicago, IL; 2020.

28. Fizazi K, Shore N, Tammela TL, Ulys A, Vjaters E, Polyakov S, et al. Overall survival (OS) results of phase III ARAMIS study of darolutamide (DARO) added to androgen deprivation therapy (ADT) for nonmetastatic castration-resistant prostate cancer (nmCRPC). American Society for Clinical Oncology: Chicago, IL; 2020.

29. Sternberg CN, Fizazi K, Saad F, Shore N, D'i Giorgi U, Penson DF, et al. Final overall survival (OS) from PROSPER: A phase III, randomized, double-blind, placebo (PBO)-controlled study of enzalutamide (ENZA) in men with nonmetastatic castrationresistant prostate cancer (nmCRPC). American Society for Clinical Oncology: Chicago, IL; 2020.

30. Pollock YG, Smith MR, Saad F, Chowdhury S, Oudard S, Hadaschik BA, et al. Predictors of falls and fractures in patients (pts) with nonmetastatic castration-resistant prostate cancer (nmCRPC) treated with apalutamide (APA) plus ongoing androgen deprivation therapy (ADT). J Clin Oncol. 2019;37:5025-5025.

31. Astellas. Xtandi ${ }^{\circledast}$ (enzalutamide). Product Insert: Northbrook IL; 2018.

32. Ryan CJ, Crawford ED, Shore ND, Underwood W, Taplin M-E, Londhe A, et al. The IMAAGEN study: effect of abiraterone acetate and prednisone on prostate specific antigen and radiographic disease progression in patients with nonmetastatic castration resistant prostate cancer. J Urol. 2018;200:344-52.

33. Kirk PS, Borza T, Shahinian VB, Caram MEV, Makarov DV, Shelton JB, et al. The implications of baseline bone-health assessment at initiation of androgen-deprivation therapy for prostate cancer. BJU Int. 2018;121:558-64.

34. Galvao DA, Noska K, Taaffe DR, Spry N, Kristjanson LJ, McGuigan MR, et al. Resistance training and reduction of treatment side effects in prostate Cancer patients. Med Sci Sports Exerc. 2006;38:2045-52.

35. Winters-Stone KM, Dobek JC, Bennett JA, Maddalozzo GF, Ryan $\mathrm{CW}$, Beer TM. Skeletal response to resistance and impact training in prostate cancer survivors. Med Sci Sports Exerc. 2014;46:1482-8.

36. Bjerre ED, Petersen TH, Jørgensen AB, Johansen C, Krustrup P, Langdahl B, et al. Community-based football in men with prostate cancer: 1-year follow-up on a pragmatic, multicentre randomised controlled trial. PLoS Med. 2019;16: e1002936-e1002936.

37. Owen PJ, Daly RM, Livingston PM, Mundell NL, Dalla Via J, Millar JL, et al. Efficacy of a multi-component exercise programme and nutritional supplementation on musculoskeletal health in men treated with androgen deprivation therapy for prostate cancer (IMPACT): study protocol of a randomised controlled trial. Trials. 2017;18:451.

38. Baron R, Ferrari S, Russell RGG. Denosumab and bisphosphonates: different mechanisms of action and effects. Bone. 2011;48:677-92.

39. Tombal BF, Loriot Y, Saad F, McDermott RS, Elliott T, RodriguezVida A, et al. Decreased fracture rate by mandating bone-protecting agents in the EORTC 1333/PEACE III trial comparing enzalutamide and Ra223 versus enzalutamide alone: an interim safety analysis. $\mathrm{J}$ Clin Oncol. 2019;37:5007-5007.

40. Poon Y, Pechlivanoglou P, Alibhai SMH, Naimark D, Hoch JS, Papadimitropoulos E, et al. Systematic review and network meta-analysis on the relative efficacy of osteoporotic medications: men with prostate cancer on continuous androgen-deprivation therapy to reduce risk of fragility fractures. BJU Int. 2018;121:17-28. 
41. Miller K, Steger GG, Niepel D, Lüftner D. Harnessing the potential of therapeutic agents to safeguard bone health in prostate cancer. Prostate Cancer Prostatic Dis. 2018;21:461-72.

42. Owen PJ, Daly RM, Livingston PM, Fraser SF. Lifestyle guidelines for managing adverse effects on bone health and body composition in men treated with androgen deprivation therapy for prostate cancer: an update. Prostate Cancer Prostatic Dis. 2017;20:137-45.

43. Fizazi K, Carducci M, Smith M, Damião R, Brown J, Karsh L, et al. Denosumab versus zoledronic acid for treatment of bone metastases in men with castration-resistant prostate cancer: a randomised, double-blind study. Lancet. 2011;377:813-22.

44. Saad F, Brown JE, Van Poznak C, Ibrahim T, Stemmer SM, Stopeck AT, et al. Incidence, risk factors, and outcomes of osteonecrosis of the jaw: integrated analysis from three blinded active-controlled phase III trials in cancer patients with bone metastases. Ann Oncol. 2012;23:1341-7.

45. Tripto-Shkolnik L, Rouach V, Marcus Y, Rotman-Pikielny P, Benbassat C, Vered I. Vertebral fractures following denosumab discontinuation in patients with prolonged exposure to bisphosphonates. Calcif Tissue Int. 2018;103:44-49.

46. Bandeira F, Torres G, Bandeira E, Duarte MB, Nobrega AM, Bandeira L. Multiple severe vertebral fractures during the 3-month period following a missed dose of denosumab in a postmenopausal woman with osteoporosis previously treated with alendronate. Int J Clin Pharmacol Ther. 2019;57:163-6.
47. Lewiecki EM. New and emerging concepts in the use of denosumab for the treatment of osteoporosis. Ther Adv Musculoskelet Dis. 2018;10:209-23.

48. Millar H, Davison J. Nutrition education for osteoporosis prevention in men with prostate cancer initiating androgen deprivation therapy. Clin J Oncol Nurs. 2012;16:497-503.

49. Alibhai SMH, Breunis H, Timilshina N, Hamidi MS, Cheung AM, Tomlinson GA, et al. Improving bone health in men with prostate cancer receiving androgen deprivation therapy: results of a randomized phase 2 trial. Cancer. 2018;124:1132-40.

50. Owen PJ, Daly RM, Dalla Via J, Mundell NL, Livingston PM, Rantalainen $\mathrm{T}$, et al. Does use of androgen deprivation therapy (ADT) in men with prostate cancer increase the risk of sarcopenia? Calcif Tissue Int. 2019;105:403-11.

51. Fizazi K, Scher HI, Miller K, Basch E, Sternberg CN, Cella D, et al. Effect of enzalutamide on time to first skeletal-related event, pain, and quality of life in men with castration-resistant prostate cancer: results from the randomised, phase 3 AFFIRM trial. Lancet Oncol. 2014;15:1147-56.

52. Medicare. Coverage for bone mass measurements. Medicare: Washington, D.C.; 2019.

53. Serpa Neto A, Tobias-Machado M, Esteves MAP, Senra MD, Wroclawski ML, Fonseca FLA, et al. Bisphosphonate therapy in patients under androgen deprivation therapy for prostate cancer: a systematic review and meta-analysis. Prostate Cancer Prostatic Dis. 2012;15:36-44. 